\title{
Vector leptoquarks beyond tree level
}

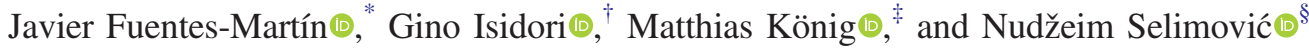 \\ Physik-Institut, Universität Zürich, CH-8057 Zürich, Switzerland
}

(Received 4 November 2019; accepted 31 January 2020; published 19 February 2020)

\begin{abstract}
Models with massive vector leptoquarks, resulting from an $S U(4)$ gauge symmetry spontaneously broken at the $\mathrm{TeV}$ scale, are of great phenomenological interest given the current "anomalies" in semileptonic $B$ decays. We analyze the relations between low- and high-energy observables in such class of models to next-to-leading order accuracy in the $S U(4)$ gauge coupling $g_{4}$. For large values of $g_{4}$, motivated by recent $B$-physics data, one-loop corrections are sizable. The main effect is an enhanced contribution at low energy at fixed on-shell couplings. This result has important implications for current and future highenergy searches of vector leptoquark models.
\end{abstract}

DOI: 10.1103/PhysRevD.101.035024

\section{INTRODUCTION}

A natural expectation of grand-unified theories, where a single fermion representation contains both quark and lepton fields, is the presence of massive vector leptoquarks, i.e., vector fields transforming quarks into leptons and vice versa. One of the most appealing constructions of this type is the model proposed by Pati and Salam (PS) [1], where quarks and leptons are unified in fundamental representations of the $S U(4)$ gauge group. The breaking $S U(4) \rightarrow$ $S U(3)_{c} \times U(1)$ gives rise to a single vector leptoquark, $U_{1}$, transforming as $(\mathbf{3}, \mathbf{1}, 2 / 3)$ under the Standard Model (SM) gauge symmetry.

A renewed phenomenological interest in the PS model has been triggered by the recent $B$-physics anomalies, i.e., the hints of lepton flavor universality violations in semileptonic $B$ decays [2-7]. Already in the early phenomenological attempts to explain these anomalies [8-11], it appeared that a $\mathrm{TeV}$-scale $U_{1}$ field, coupled mainly to the third generation, is an excellent mediator to account for all available data.

The problem of the original PS model in this context is the flavor-universal nature of the $U_{1}$, which has to be very heavy in order to satisfy the tight bounds derived from its coupling to light SM fermions. This problem can be overcome in a natural way with two main ingredients: enlarging the gauge group [12] and allowing gauge nonuniversal charges to the

\footnotetext{
*fuentes@physik.uzh.ch

isidori@physik.uzh.ch

*matthias.koenig@uzh.ch

${ }^{\S}$ nudzeim@physik.uzh.ch
}

Published by the American Physical Society under the terms of the Creative Commons Attribution 4.0 International license. Further distribution of this work must maintain attribution to the author(s) and the published article's title, journal citation, and DOI. Funded by SCOAP ${ }^{3}$.
SM fermions [13]. These two ingredients have been analyzed in a series of recent papers [12-16]. The proposed models have a few differences, but the TeV-scale dynamics is always characterized by the gauge group $S U(4) \times$ $S U(3) \times S U(2) \times U(1)$, effectively acting in a family nonuniversal way. These models, which we collectively denote as 4321 models, provide both a successful ultraviolet (UV) completion for effective descriptions of the $B$-physics anomalies and, at the same time, represent a first step to shed light on the origin of SM mass hierarchies [13] (alternative approaches to embed the $U_{1}$ in extended PS-type models have been proposed in $[17,18]$ ).

The spontaneous symmetry breaking of the 4321 gauge group to $G_{\mathrm{SM}}=S U(3)_{c} \times S U(2)_{L} \times U(1)_{Y}$ gives rise to two additional massive vectors beside the $U_{1}$ : a color octet $G^{\prime} \sim(\mathbf{8}, \mathbf{1}, 0)$, commonly referred to as coloron, and a color singlet $Z^{\prime} \sim(\mathbf{1}, \mathbf{1}, 0)$. As pointed out in [19], the presence of (at least) these additional states is a general feature of any UV completion of a flavor nonuniversal $U_{1}$. These states are indeed present also in UV completions based on new strongly interacting dynamics [20,21].

So far, the dynamics of these heavy vectors has been analyzed only at leading order in the leptoquark (LQ) coupling. Next-to-leading order (NLO) effects in QCD have been studied, both at low energies [22] (in the corrections to the coefficients of the corresponding four-fermion operators) and at high energies [23] (in LQ production and decay at colliders). However, NLO corrections associated to the heavy dynamics have never been analyzed. In the absence of a UV completion, neglecting these corrections is a necessary choice. But the validity of this approximation is questionable given that the coupling of the leptoquark to SM fermions must be large $\left(2 \lesssim g_{4} \lesssim 3\right)$ in order to explain $B$-physics data, while being consistent with collider searches.

Employing a simplified 4321 model, which provides a consistent and sufficiently general description of the 
heavy-vector dynamics, we present, for the first time, an estimate of the NLO corrections associated to the leptoquark coupling $\left(g_{4}\right)$. Since the latter is large, we work in the limit where all SM couplings (both gauge and Yukawa) are set to zero. This limit simplifies the calculation and isolates all the leading effects proportional to $\alpha_{4}=g_{4}^{2} /(4 \pi)$, without loss of generality. The results obtained this way are applicable to all the realistic 4321 models proposed in the literature. Being interested only in the physical effects generated by these quantum corrections, we adopt an on-shell renormalization scheme: masses and couplings of the heavy states are defined from their on-shell production and decay processes. We evaluate NLO corrections to low-energy amplitudes in terms of these parameters to perform the matching to the Standard Model Effective Theory (SMEFT).

\section{THE MODEL}

We consider a simplified version of the 4321 model, where we set the SM gauge couplings to zero, $g_{1,2,3}=0$. Furthermore, we ignore the SM Higgs sector, meaning that the model has an exact $S U(3)_{12} \times S U(2)_{L} \times U(1)_{Y}$ global symmetry: the $S U(3)_{12}$ group acts only on the light generations, which decouple being $S U(4)$ singlets. The $S U(2)_{L} \times U(1)_{Y}$ group is flavor universal. The only nontrivial dynamics is that of the $S U(4)$ gauge group, with coupling $g_{4}$.

The nondecoupling fermion fields are one $S U(2)_{L}$ doublet, $\psi_{L}$, and two $S U(2)_{L}$ singlets, $\psi_{u}$ and $\psi_{d}$. As we discuss later, these fields can be identified with the SM third generation, up to (small) mixings with the light families and/or mixings with heavy exotic fermions. In the SM-gaugeless limit, these massless fields consist of two identical vectorlike fermions transforming in the fundamental representation of $S U(4)$.

The spontaneous breaking of $S U(4)$ is achieved by two $S U$ (4)-fundamental scalars, $\Omega_{1}$ and $\Omega_{3}$, transforming as singlet and triplet under $S U(3)$, respectively. The Lagrangian of this simplified model reads

$$
\begin{aligned}
\mathcal{L}= & -\frac{1}{4} H_{\mu \nu}^{\alpha} H^{\alpha \mu \nu}+\sum_{i=1,3}\left(D_{\mu} \Omega_{i}\right)^{\dagger} D_{\mu} \Omega_{i} \\
& +\sum_{f=L, u, d} i \bar{\psi}_{f} \not D \psi_{f}+V\left(\Omega_{i}\right),
\end{aligned}
$$

where $H_{\mu \nu}^{\alpha}(\alpha=1, \ldots, 15)$ is the $S U(4)$ field-strength tensor. We further assume that all the radial modes are much heavier than the vector resonances $\left(M_{R}^{2} \gg g_{4}^{2} v_{3(1)}^{2}\right)$, with $v_{1(3)}$ denoting the vacuum expectation of $\Omega_{1}\left(\Omega_{3}\right)$. This way, we can restrict the attention to the dynamics of gauge fields, Goldstone bosons, and fermions. ${ }^{1}$

\footnotetext{
${ }^{1}$ An extended analysis including radial modes and heavy fermions will be presented elsewhere [24].
}

After spontaneous symmetry breaking, no physical scalars remain massless and all $S U(4)$ gauge fields acquire a mass. The latter can be identified with the massive vector resonances of the realistic 4321 models [12-16]. The charge and mass eigenstates of the $S U(4)$ gauge bosons $H^{\alpha}$ are

$$
\begin{aligned}
G_{\mu}^{\prime} & =H_{\mu}^{a}, \quad Z_{\mu}^{\prime}=H_{\mu}^{15}, \\
U_{\mu}^{1,2,3} & =\frac{1}{\sqrt{2}}\left(H_{\mu}^{9,11,13}-i H_{\mu}^{10,12,14}\right),
\end{aligned}
$$

with masses $m_{G^{\prime}}^{2}=\left(g_{4}^{2} / 2\right) v_{3}^{2}, m_{U}^{2}=\left(g_{4}^{2} / 4\right)\left(v_{1}^{2}+v_{3}^{2}\right)$ and $m_{Z^{\prime}}^{2}=\left(3 g_{4}^{2} / 8\right)\left(v_{1}^{2}+v_{3}^{2} / 3\right)$. In the limit $v_{1}=v_{3}$, there is a residual custodial $S U(4)$ global symmetry and all massive vectors are degenerate.

In the mass eigenbasis, the interactions between vectors and fermions read

$$
\begin{aligned}
\mathcal{L}_{\text {int }} \supset & \frac{g_{4}}{\sqrt{2}}\left[U_{\mu} \bar{\psi}_{q} \gamma^{\mu} \psi_{\ell}+\text { H.c. }\right]+g_{4} G_{\mu}^{\prime} \bar{\psi}_{q} \gamma^{\mu} T^{a} \psi_{q} \\
& +\frac{g_{4} \sqrt{6}}{4} Z_{\mu}^{\prime}\left(\bar{\psi} T_{\mathrm{B}-\mathrm{L}} \gamma^{\mu} \psi\right),
\end{aligned}
$$

where $\psi=\left(\psi_{q} \psi_{\ell}\right)^{\top}$ are $S U(4)$ fermion multiplets and $T_{\mathrm{B}-\mathrm{L}}=\operatorname{diag}\left(\frac{1}{3}, \frac{1}{3}, \frac{1}{3},-1\right)$.

\section{ONE-LOOP RESULTS}

Our simplified model is completely renormalizable only after the inclusion of the radial modes; however, this does not prevent us from obtaining finite and gauge-invariant results in the on-shell scheme, once we add an appropriate set of counterterms (as in the nonlinear sigma model). The results obtained this way are correct up to finite terms of $O\left(m_{V}^{2} / M_{R}^{2}\right)$ which we assume to be small. The explicit inclusion of the Goldstone modes ensures gauge-invariant results. All partial results reported below are obtained in the Feynman gauge.

\section{A. Vertex corrections}

We start analyzing the correction to the three-point functions with one external heavy vector and two light fermions. The modified LQ vertex function assumes the form

$$
\mathcal{A}_{\text {vertex }}^{U}=i \frac{g_{4}}{\sqrt{2}} \epsilon_{\mu}(q) \psi_{q} \gamma^{\mu} \psi_{\ell} \times\left[1+\frac{\alpha_{4}}{4 \pi} \delta V_{U}(s)\right],
$$

where $\epsilon_{\mu}(q)$ is the LQ polarization vector and $s=q^{2}$. Using dimensional regularization in $d=4-2 \epsilon$, we find

$\delta V_{U}(s)=\frac{47}{8}\left(\frac{1}{\epsilon}+\log \frac{\mu^{2}}{m_{U}^{2}}\right)+\Lambda_{U}^{0}+\Lambda_{U}\left(s,\left\{m_{V_{i}}^{2}\right\}\right)$, 
where $\Lambda_{U}^{0}$ is constant and the $s$-dependent term, satisfying $\Lambda_{U}\left(0,\left\{m_{V_{i}}^{2}\right\}\right)=0$, can be expressed as

$$
\begin{aligned}
\Lambda_{U}\left(s,\left\{m_{V_{i}}^{2}\right\}\right)= & -\frac{1}{8} \Lambda_{2}\left(s, m_{Z^{\prime}}\right)+2 \Lambda_{4}\left(s, m_{Z^{\prime}}, m_{U}\right) \\
& +4 \Lambda_{4}\left(s, m_{G^{\prime}}, m_{U}\right)
\end{aligned}
$$

in terms of the loop function reported in [25]. Note that the coefficient of the UV divergence is nothing but $C_{A}+C_{F}$. After renormalization, defining the renormalized coupling from the on-shell LQ vertex, the finite vertex correction (for off-shell processes) reads

$$
\delta V_{U}(s)_{r}=\delta V_{U}(s)-\operatorname{Re}\left[\delta V_{U}\left(m_{U}^{2}\right)\right]
$$

Under these renormalization conditions, the constant terms in (5) do not play a role in physical observables. At $s=0$, and in the $S U(4)$ custodial limit for the vector-boson masses, we find

$$
\delta V_{U}(0)_{r}^{(\text {cust })}=-\frac{27}{16}+\frac{17}{12} \pi^{2}-2 \sqrt{3} \pi \approx 1.41
$$

Proceeding in a similar way for the coloron and $Z^{\prime}$ vertices, we find an identical UV divergence, the following $s$-dependent terms:

$$
\begin{aligned}
\Lambda_{G^{\prime}}\left(s,\left\{m_{V_{i}}^{2}\right\}\right)= & \frac{1}{24} \Lambda_{2}\left(s, m_{Z^{\prime}}\right)-\frac{1}{6} \Lambda_{2}\left(s, m_{G^{\prime}}\right) \\
& +\frac{3}{2} \Lambda_{4}\left(s, m_{U}, m_{U}\right)+\frac{9}{2} \Lambda_{4}\left(s, m_{G^{\prime}}, m_{G^{\prime}}\right),
\end{aligned}
$$

$$
\begin{aligned}
\Lambda_{Z^{\prime}}\left(s,\left\{m_{V_{i}}^{2}\right\}\right)= & \frac{7}{24} \Lambda_{2}\left(s, m_{Z^{\prime}}\right)+\frac{1}{3} \Lambda_{2}\left(s, m_{G^{\prime}}\right) \\
& -\frac{3}{4} \Lambda_{2}\left(s, m_{U}\right)+6 \Lambda_{4}\left(s, m_{U}, m_{U}\right) \\
& +T_{\mathrm{B}-\mathrm{L}}^{-1}\left[\frac{1}{3} \Lambda_{2}\left(s, m_{G^{\prime}}\right)\right. \\
& \left.-\frac{1}{12} \Lambda_{2}\left(s, m_{Z^{\prime}}\right)-\frac{1}{4} \Lambda_{2}\left(s, m_{U}\right)\right]
\end{aligned}
$$

and the following constant terms:

$$
\begin{aligned}
\Lambda_{G^{\prime}}^{0}-\Lambda_{U}^{0}= & -6+\left(\frac{4 x_{G^{\prime}}}{x_{G^{\prime}}-1}-\frac{9}{2}\right) \log \left(x_{G^{\prime}}\right) \\
& +\left(\frac{2 x_{Z^{\prime}}}{x_{Z^{\prime}}-1}-\frac{1}{6}\right) \log \left(x_{Z^{\prime}}\right) \stackrel{x_{\mathrm{V}}=1}{\longrightarrow} 0,
\end{aligned}
$$

$$
\begin{aligned}
\Lambda_{Z^{\prime}}^{0}-\Lambda_{U}^{0}= & -6+\left(\frac{4 x_{G^{\prime}}}{x_{G^{\prime}}-1}-\frac{1}{3}\right) \log \left(x_{G^{\prime}}\right) \\
& +\left(\frac{2 x_{Z^{\prime}}}{x_{Z^{\prime}}-1}-\frac{5}{12}\right) \log \left(x_{Z^{\prime}}\right) \\
& +T_{\mathrm{B}-\mathrm{L}}^{-1}\left[\frac{1}{12} \log \left(x_{Z^{\prime}}\right)-\frac{1}{3} \log \left(x_{G^{\prime}}\right)\right] \stackrel{x_{\mathrm{V}}=1}{\longrightarrow} 0,
\end{aligned}
$$

with $x_{V}=m_{V}^{2} / m_{U}^{2}$. After renormalization,

$$
\delta V_{V}(s)_{r}=\delta V_{V}(s)-\operatorname{Re}\left[\delta V_{U}\left(m_{U}^{2}\right)\right] .
$$

In general, both vertex functions $\left(V=G^{\prime}, Z^{\prime}\right)$ are nonvanishing in the on-shell case (i.e., for $s=m_{V}^{2}$ ). However, as shown by the $x_{V} \rightarrow 1$ limits, they do vanish on shell in the $S U(4)$ custodial limit.

\section{B. Two-point functions}

The LQ propagator in the Feynman gauge, corrected by resumming one-particle reducible diagrams, can be written as

$$
\frac{-i g^{\mu \nu}}{p^{2}-m_{U}^{2}}\left[1+\frac{\alpha_{4}}{4 \pi} \delta \Sigma_{U}\left(p^{2}\right)\right]+\mathcal{O}\left(p^{\mu} p^{\nu}\right)
$$

where we have already expressed the result in terms of the renormalized mass, and we have taken into account the wave-function renormalization. The nontrivial corrections are encoded in the finite term $\delta \Sigma_{U}(s)$ that we can express in the on-shell scheme as

$$
\delta \Sigma_{U}(s)=\frac{\Sigma_{U}(s)-\Sigma_{U}\left(m_{U}^{2}\right)}{s-m_{U}^{2}}-\left.\frac{\partial \Sigma_{U}(s)}{\partial s}\right|_{s=m_{U}^{2}}
$$

in terms of the reduced self-energy function $\Sigma_{U}(s)$. The explicit one-loop calculation yields

$$
\begin{aligned}
\Sigma_{U}(s)= & \Sigma_{U}^{0}+s \Sigma_{U}^{1}+\frac{N_{f}}{3} s \log \left(-\frac{s}{m_{U}^{2}}\right) \\
& +\left[\frac{m_{U}^{4}}{s}\left(-\frac{x_{G^{\prime}}^{3}}{9}-\frac{2 x_{G^{\prime}}^{2}}{3}+\frac{5 x_{G^{\prime}}}{3}-\frac{8}{9}\right)-s\left(\frac{x_{G^{\prime}}}{9}-\frac{40}{9}\right)\right. \\
& \left.+m_{U}^{2}\left(\frac{16}{9}+6 x_{G^{\prime}}-\frac{10 x_{G^{\prime}}^{2}}{9}\right)\right] F\left(s, m_{U}^{2}, m_{G^{\prime}}^{2}\right) \\
& +\left[\frac{m_{U}^{4}}{s}\left(-\frac{x_{Z^{\prime}}^{3}}{18}-\frac{x_{Z^{\prime}}^{2}}{3}+\frac{5 x_{Z^{\prime}}}{6}-\frac{4}{9}\right)-s\left(\frac{x_{Z^{\prime}}}{18}-\frac{20}{9}\right)\right. \\
& \left.+m_{U}^{2}\left(\frac{8}{9}+3 x_{Z^{\prime}}-\frac{5 x_{Z^{\prime}}^{2}}{9}\right)\right] F\left(s, m_{U}^{2}, m_{Z^{\prime}}^{2}\right) .
\end{aligned}
$$

Here $\Sigma_{U}^{0}$ and $\Sigma_{U}^{1}$ are constant divergent terms, absorbed by the renormalization procedure; $N_{f}$ denotes the number of 
TABLE I. Coefficients of the semileptonic operators, normalized as in (19), at tree level and NLO (box contributions only). The NLO results are in units of $\alpha_{4} /(4 \pi), x_{V}=m_{V}^{2} / m_{U}^{2}$, and $f_{V}=\log \left(x_{V}\right) /\left(x_{V}-1\right)$.

\begin{tabular}{|c|c|c|c|c|c|c|c|}
\hline \multirow[b]{2}{*}{ Operators } & \multicolumn{2}{|c|}{ Tree level } & \multicolumn{5}{|c|}{ NLO box contributions (in units of $\frac{\alpha_{4}}{4 \pi}$ ) } \\
\hline & $U_{1}$ & $Z^{\prime}$ & {$\left[G^{\prime} U_{1}\right]$} & {$\left[Z^{\prime} U_{1}\right]$} & {$\left[U_{1} U_{1}\right]$} & {$\left[Z^{\prime} Z^{\prime}\right]$} & Total $\left(x_{V}=1\right.$ limit $)$ \\
\hline $\mathcal{O}_{L L}^{U}=\frac{1}{2}\left(\mathcal{O}_{\ell q}^{(1)}+\mathcal{O}_{\ell q}^{(3)}\right)$ & 1 & 0 & $\frac{4}{3} f_{G^{\prime}}$ & $\frac{17}{12} f_{Z^{\prime}}$ & & & $+\frac{11}{4}$ \\
\hline $\mathcal{O}_{L R}^{U}=-2 \mathcal{O}_{\ell e d q}$ & 1 & 0 & $\frac{16}{3} f_{G^{\prime}}$ & $\frac{23}{12} f_{Z^{\prime}}$ & & & $+\frac{29}{4}$ \\
\hline $\mathcal{O}_{\ell q}^{(1)}$ & 0 & $-\frac{1}{4 x_{z^{\prime}}}$ & & & -2 & $-\frac{3}{32 x_{Z^{\prime}}}$ & $-\frac{67}{32}$ \\
\hline $\mathcal{O}_{\ell d}$ & 0 & $-\frac{1}{4 x_{z^{\prime}}}$ & & & $-\frac{1}{2}$ & $\frac{3}{32 x_{Z^{\prime}}}$ & $-\frac{13}{32}$ \\
\hline $\mathcal{O}_{q e}$ & 0 & $-\frac{1}{4 x_{z^{\prime}}}$ & & & $-\frac{1}{2}$ & $\frac{3}{32 x_{z^{\prime}}}$ & $-\frac{13}{32}$ \\
\hline $\mathcal{O}_{d e}$ & 1 & $-\frac{1}{4 x_{z^{\prime}}}$ & $\frac{4}{3} f_{G^{\prime}}$ & $\frac{17}{12} f_{Z^{\prime}}$ & -2 & $-\frac{3}{32 x_{Z^{\prime}}}$ & $+\frac{21}{32}$ \\
\hline
\end{tabular}

light fermion species transforming in the fundamental of $S U(4)$; while $F\left(s, m_{X}^{2}, m_{Y}^{2}\right)$, defined as in [25], satisfies $F\left(0, m_{X}^{2}, m_{Y}^{2}\right)=0$.

The finite correction to the two-point function at $s=0$ assumes the following value in the custodial limit:

$$
\delta \Sigma_{U}(0)^{(\text {cust })}=\frac{73}{2}-7 \sqrt{3} \pi-\frac{N_{f}}{3} \stackrel{N_{f}=4}{\longrightarrow} \approx-2.92 .
$$

Combining the results of two- and three-point functions, we can evaluate the overall NLO correction induced by one-particle reducible diagrams to the LQ-mediated fourfermion amplitude at low energies,

$$
\mathcal{A}_{1 \mathrm{P}-\mathrm{red}}^{\mathrm{NLO}}=\mathcal{A}^{\text {tree }}\left\{1+\frac{\alpha_{4}}{4 \pi}\left[\delta \Sigma_{U}(0)+2 \delta V_{U}(0)_{r}\right]\right\} .
$$

This correction turns out to be very small: in the custodial limit, the two terms cancel to a remarkable accuracy, resulting in a correction below $1 \%$ (in absolute size) even for $g_{4}=3$. More precisely, setting $g_{4}=3$ and $N_{f}=4,{ }^{2}$ the correction lies between $-1 \%$ and 0 for $x_{G^{\prime}}>0.7$. Sizable positive values can be obtained only for small $x_{G^{\prime}}$ values, but the correction does not exceed $1 \%$ for $x_{G^{\prime}}>0.5$.

The smallness of this NLO correction can be understood as a consequence of the sudden stop in the running of $\alpha_{4}$ below the LQ mass, when employing a physical (massdependent) renormalization procedure. The one-particle reducible diagrams are indeed responsible for the running of $\alpha_{4}$ and their combined effect turns out to be particularly small in the custodial limit, where all the heavy particles decouple together, at the scale used to define the renormalized coupling.

We have checked that a similar cancellation holds also for one-particle reducible contributions to coloron- and $Z^{\prime}$ mediated four-fermion amplitudes. The complete expressions for the corresponding self-energy functions, $\Sigma_{G^{\prime}, Z^{\prime}}(s)$, which coincide with $\Sigma_{U}(s)$ in the custodial limit, will be reported elsewhere [24].

\footnotetext{
${ }^{2}$ The value $N_{f}=4(3)$ corresponds to the case where we treat the right-handed neutrino mass as light (heavy) compared to $m_{U}$.
}

\section{Box diagrams and matching onto the SMEFT}

Due to the effective cancellation of one-particle reducible contributions, the only potentially large NLO effects in four-fermion processes originate from box diagrams.

The result of the box diagrams in the limit of vanishing external momenta can be matched onto the basis of dimension-six SMEFT operators [26]. Normalizing the Lagrangian as

$$
\mathcal{L}_{\mathrm{SMEFT}}=-\frac{g_{4}^{2}}{2 M_{U}^{2}} \sum_{k} \mathcal{C}_{k} \mathcal{O}_{k},
$$

the Wilson coefficients for the relevant semileptonic operators are reported in Table I. To better illustrate the result, we perform a change of basis compared to [26] introducing the combinations

$$
\begin{aligned}
& \mathcal{O}_{L L}^{U}=\left(\bar{\ell}_{L} \gamma_{\mu} q_{L}\right)\left(\bar{q}_{L} \gamma^{\mu} l_{L}\right) \\
& \mathcal{O}_{L R}^{U}=-2\left(\bar{\ell}_{L} e_{R}\right)\left(\bar{d}_{R} q_{L}\right)+\text { H.c. },
\end{aligned}
$$

which, at the tree level, are the only effective operators generated by the LQ exchange.

Our simplified model features only a single fermion family; hence, there is no flavor mixing. However, results for realistic models addressing the $B$-physics anomalies can be recovered assuming a specific direction for this family in flavor space (switching on the Yukawa couplings) and/or introducing appropriate nontrivial flavor structures in the currents in Eq. (3), resulting from mixing with heavy fermions. This way it is easy to realize that $\mathcal{O}_{L L}^{U}$ is the lefthanded operator contributing to $b \rightarrow c \tau \nu$, which is present in all the 4321 models, whereas $\mathcal{O}_{L R}^{U}$ is the scalar operator present in models where the $U_{1}$ has also right-handed couplings $[13,16]$. As shown in Table $\mathrm{I}$, in the custodial limit, we find a $16 \%(41 \%)$ enhancement for $\mathcal{C}_{L L}^{U}\left(\mathcal{C}_{L R}^{U}\right)$ at NLO, at fixed on-shell coupling $g_{4}=3$.

We stress that the effects we have estimated are only due to the new dynamics of the heavy vectors; therefore, they should be considered in addition to the QCD corrections to 

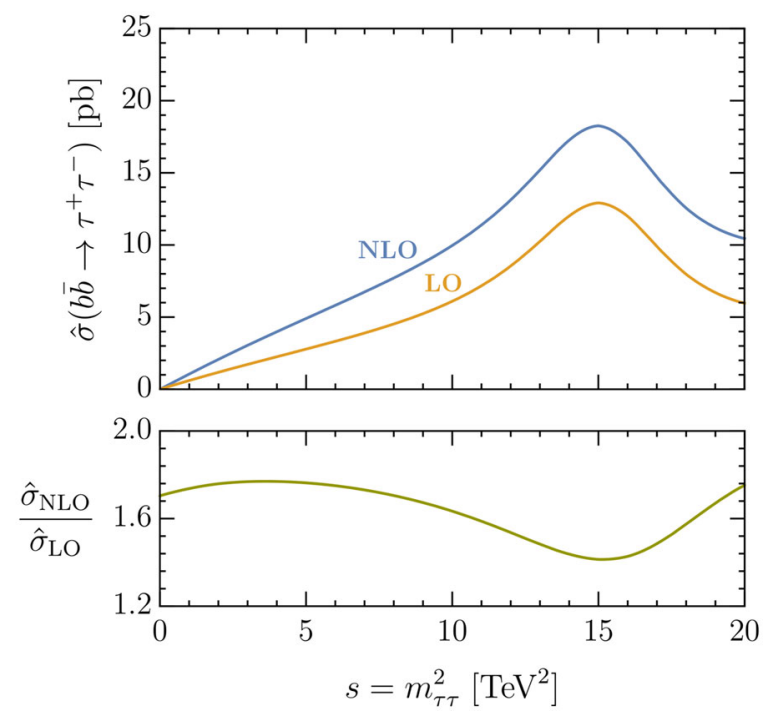

FIG. 1. Upper panel: $b \bar{b} \rightarrow \tau^{+} \tau^{-}$partonic cross section at LO and NLO, as a function of $s=m_{\tau \tau}^{2}$. Lower panel: NLO/LO ratio.

the high-scale matching conditions estimated in [22]. According to this recent analysis, the $\mathcal{O}\left(\alpha_{s}\right)$ corrections to $\mathcal{C}_{L L}^{U}$ and $\mathcal{C}_{L R}^{U}$ go in the same direction of the $\mathcal{O}\left(\alpha_{4}\right)$ ones, i.e., they enhance the coefficients of the effective operators, and are comparable (significantly smaller) with respect to the $\mathcal{O}\left(\alpha_{4}\right)$ terms in the case of $\mathcal{C}_{L L}^{U}\left(\mathcal{C}_{L R}^{U}\right)$.

Our findings have important phenomenological consequences: they imply that all collider bounds dominated by the on-shell $s$-channel production of the new states (i.e., the single production of coloron, $Z^{\prime}$, and leptoquark) are significantly weaker at fixed low-energy contribution. This suppression holds only for the on-shell production of the resonances where (i) the cross section can be expressed in terms of the on-shell renormalized couplings, (ii) the contribution of the box amplitudes is subleading (being nonresonant). This is illustrated in Fig. 1, where we show the $b \bar{b} \rightarrow \tau \bar{\tau}$ partonic cross section at LO and NLO in $\alpha_{4}$, within the 4321 model of Ref. [13], setting $g_{4}=3$ and $v_{1}=v_{3}$ such that $M_{V}=4 \mathrm{TeV}$. The NLO result takes into account all contributions ( $s$-channel $Z^{\prime}, t$-channel $U_{1}$, and box amplitudes-with full kinematical dependence). As can be seen, NLO corrections amount to a large increase compared to the LO result. Far from the resonant region, the effect is quite similar to the pure low-energy (SMEFT) regime. On the other hand, the enhancement becomes smaller close to the $Z^{\prime}$ peak, where the process is dominated by the on-shell contribution. The suppression is stronger in processes where the resonant amplitude is larger, such as $g b \rightarrow U_{1} \tau \rightarrow(b \bar{\tau}) \tau$, dominated by the $U_{1}$ exchange.
Another important phenomenological implication of Table I is the large NLO correction to the coefficient of the singlet operator $\mathcal{O}_{\ell q}^{(1)}$. At tree level, this operator is not generated by $U_{1}$ exchange, ${ }^{3}$ allowing the model to evade the strong experimental bounds on $b \rightarrow s \nu_{\tau} \bar{\nu}_{\tau}$ and $s \rightarrow d \nu_{\tau} \bar{\nu}_{\tau}$ transitions [9-11]. As shown in Table I, this operator necessarily appears at the one-loop level, even considering only box diagrams with leptoquarks. The latter lead to an NLO coefficient for $\mathcal{O}_{\ell q}^{(1)}$ which is $11 \%$ of the LO contribution to $\mathcal{O}_{L L}^{U}$ for $g_{4}=3$. Currently, this does not pose a serious problem for $U_{1}$ models addressing the $B$-physics anomalies. However, it implies that in $\mathcal{B}(B \rightarrow$ $\left.K^{(*)} \nu \nu\right)$ and $\mathcal{B}(K \rightarrow \pi \nu \nu)$ one should expect $O(10 \%-$ $100 \%)$ modifications compared to the corresponding SM predictions (see, e.g., [11,27]).

\section{CONCLUSIONS}

$\mathrm{TeV}$-scale vector leptoquarks are currently the subject of numerous experimental investigations, both at low and at high energies. If the LQ coupling to SM fermions is large, as expected in motivated models addressing the $B$-physics anomalies, potentially large effects beyond tree level should be expected. In this paper, we have presented the first estimate of these effects in a general class of models based on extensions of the PS gauge symmetry. As expected, NLO corrections are large, but they are calculable and still within a perturbative regime for $g_{4} \lesssim 3$. The main effect is an enhanced LQ contribution at low energy, at fixed onshell couplings. This implies weaker constraints from highenergy (on shell) LQ searches in realistic models addressing $B$-physics anomalies.

\section{ACKNOWLEDGMENTS}

We thank Sandro Mächler for collaboration in the early stage of this project. We are also grateful to Admir Greljo for useful comments. This project received funding from the European Research Council under the European Union's Horizon 2020 research and innovation programme under Grant No. 833280 (FLAY) and by the Swiss National Science Foundation under Contract No. 200021-159720. The work of J.F. was also supported in part by the Generalitat Valenciana under Contract No. SEJI/2018/033.

\footnotetext{
${ }^{3}$ A potentially dangerous tree-level contribution to $\mathcal{O}_{\ell q}^{(1)}$ is generated by $Z^{\prime}$ exchange. However, the flavor-violating component of the latter can be suppressed choosing a different flavormixing structure for quark-quark and quark-lepton currents [15].
} 
[1] J. C. Pati and A. Salam, Phys. Rev. D 10, 275 (1974); 11, 703(E) (1975).

[2] R. Aaij et al. (LHCb Collaboration), Phys. Rev. D 97, 072013 (2018).

[3] S. Hirose et al. (Belle Collaboration), Phys. Rev. Lett. 118, 211801 (2017).

[4] R. Aaij et al. (LHCb Collaboration), Phys. Rev. Lett. 115, 111803 (2015); 115, 159901(E) (2015).

[5] J. P. Lees et al. (BABAR Collaboration), Phys. Rev. D 88, 072012 (2013).

[6] R. Aaij et al. (LHCb Collaboration), Phys. Rev. Lett. 113, 151601 (2014).

[7] R. Aaij et al. (LHCb Collaboration), J. High Energy Phys. 08 (2017) 055.

[8] R. Alonso, B. Grinstein, and J. M. Camalich, J. High Energy Phys. 10 (2015) 184.

[9] L. Calibbi, A. Crivellin, and T. Ota, Phys. Rev. Lett. 115, 181801 (2015).

[10] R. Barbieri, G. Isidori, A. Pattori, and F. Senia, Eur. Phys. J. C 76, 67 (2016).

[11] D. Buttazzo, A. Greljo, G. Isidori, and D. Marzocca, J. High Energy Phys. 11 (2017) 044.

[12] L. Di Luzio, A. Greljo, and M. Nardecchia, Phys. Rev. D 96, 115011 (2017).

[13] M. Bordone, C. Cornella, J. Fuentes-Martín, and G. Isidori, Phys. Lett. B 779, 317 (2018).
[14] A. Greljo and B. A. Stefanek, Phys. Lett. B 782, 131 (2018).

[15] L. Di Luzio, J. Fuentes-Martín, A. Greljo, M. Nardecchia, and S. Renner, J. High Energy Phys. 11 (2018) 081.

[16] C. Cornella, J. Fuentes-Martín, and G. Isidori, J. High Energy Phys. 07 (2019) 168.

[17] N. Assad, B. Fornal, and B. Grinstein, Phys. Lett. B 777, 324 (2018).

[18] L. Calibbi, A. Crivellin, and T. Li, Phys. Rev. D 98, 115002 (2018).

[19] M. J. Baker, J. Fuentes-Martín, G. Isidori, and M. König, Eur. Phys. J. C 79, 334 (2019).

[20] R. Barbieri and A. Tesi, Eur. Phys. J. C 78, 193 (2018).

[21] M. Blanke and A. Crivellin, Phys. Rev. Lett. 121, 011801 (2018).

[22] J. Aebischer, A. Crivellin, and C. Greub, Phys. Rev. D 99, 055002 (2019).

[23] J. B. Hammett and D. A. Ross, J. High Energy Phys. 07 (2015) 148.

[24] J. Fuentes-Martín, G. Isidori, M. König, and N. Selimović (to be published).

[25] M. Böhm, H. Spiesberger, and W. Hollik, Fortschr. Phys. 34, 687 (1986).

[26] B. Grzadkowski, M. Iskrzynski, M. Misiak, and J. Rosiek, J. High Energy Phys. 10 (2010) 085.

[27] M. Bordone, D. Buttazzo, G. Isidori, and J. Monnard, Eur. Phys. J. C 77, 618 (2017). 